\title{
Augmented reality through head-mounted display for navigation of baseplate component placement in reverse total shoulder arthroplasty: a cadaveric study
}

\author{
Philipp Kriechling $^{1}{ }^{10} \cdot$ Rafael Loucas $^{1} \cdot$ Marios Loucas $^{1} \cdot$ Fabio Casari $^{1} \cdot$ Philipp Fürnstahl $^{2} \cdot$ Karl Wieser $^{1}$
}

Received: 4 January 2021 / Accepted: 23 June 2021 / Published online: 2 July 2021

(c) The Author(s) 2021

\begin{abstract}
Background To achieve an optimal clinical outcome in reverse total shoulder arthroplasty (RSA), accurate placement of the components is essential. The recently introduced navigation technology of augmented reality (AR) through head-mounted displays (HMD) offers a promising new approach to visualize the anatomy and navigate component positioning in various orthopedic surgeries. We hypothesized that AR through HMD is feasible, reliable, and accurate for guidewire placement in RSA baseplate positioning.

Methods Twelve human cadaver shoulders were scanned with computed tomography (CT) and RSA baseplate positioning was 3-D planned using dedicated software. The shoulders were prepared through a deltopectoral approach and an augmented reality hologram was superimposed using the HMD Microsoft HoloLense. The central guidewire was then navigated through the HMD to achieve the planned entry point and trajectory. Postoperatively, the shoulders were CT-scanned a second time and the deviation from the planning was calculated.

Results The mean deviation of the entry point was $3.5 \mathrm{~mm} \pm 1.7 \mathrm{~mm}(95 \%$ CI $2.4 \mathrm{~mm} ; 4.6 \mathrm{~mm})$. The mean deviation of the planned trajectory was $3.8^{\circ} \pm 1.7^{\circ}\left(95 \%\right.$ CI $\left.2.6^{\circ} ; 4.9^{\circ}\right)$.

Conclusion Augmented reality seems feasible and reliable for baseplate guidewire positioning in reverse total shoulder arthroplasty. The achieved values were accurate.
\end{abstract}

Keywords Reverse total shoulder arthroplasty $\cdot$ Augmented reality $\cdot$ Head-mounted display $\cdot$ Navigation $\cdot$ Experimental Cadaveric · Orthopedic surgery

Philipp Kriechling

philipp.kriechling@balgrist.ch

Rafael Loucas

rafael.loucas@balgrist.ch

Marios Loucas

marios.loucas@hotmail.com

Fabio Casari

fabio.casari@balgrist.ch

Philipp Fürnstahl

philipp.fuernstahl@balgrist.ch

Karl Wieser

karl.wieser@balgrist.ch

1 Department of Orthopaedics, Balgrist University Hospital, Zurich, Switzerland

2 Computer Assisted Research and Development Group, Balgrist University Hospital, Zurich, Switzerland

\section{Introduction}

The use of reverse total shoulder arthroplasty (RSA) is spreading worldwide due to an aging population and an increasing variety of indications. Initially, RSA was indicated in patients suffering from rotator cuff arthropathy, but nowadays, RSA has become a valid option for massive rotator cuff tear, osteoarthritis, primary fracture treatment, or revision surgery [1-3]. To achieve optimal and reliable component placement, the implantation systems and techniques are being continuously improved. The optimal baseplate position is considered in a neutral version and neutral to slightly inferior inclination [4-6]. Especially in cases of severe glenoid deformation or complex revision surgeries, the accurate position of the baseplate can, however, be challenging due to bone deficiency and limited intraoperative visibility. Malpositioning of the components can potentially lead to complications like inferior scapular 
notching, loosening or instability, and unsatisfying clinical outcomes [7].

Computer-assisted pose tracking navigation or patientspecific instrumentation can be utilized to achieve the planned baseplate position. Both systems offer promising results but have a couple of disadvantages. Navigation systems are expensive to purchase and can be uncomfortable to use, potentially increasing surgical time. Patientspecific guides require a particular time of preoperative manufacturing and intraoperative specific handling $[8$, 9]. Recently, the technology of augmented reality (AR) through a head-mounted display (HMD) has been introduced [10]. This technology seems easy to use and comparably cheap without the disadvantage of long preoperative preparation time. The current literature on this topic is very sparse, especially regarding shoulder surgery. Berhouet et al. [11] described the 3D projection of a hologram of a reconstructed scapula. Gregory et al. [12] presented the intraoperative projection in one case. Both did not utilize the HMD for navigation but could prove the high potential of AR in RSA surgery. In a previous study, we analyzed the feasibility of HMD in ten 3D-printed bone models and found a mean deviation from the entry point of $2.26 \mathrm{~mm} \pm 1.11 \mathrm{~mm}$. The deviation from the planned vector was $2.74^{\circ} \pm 1.25^{\circ}[13]$.

This study aimed to investigate the feasibility of AR navigation through HMD to guide the RSA baseplate positioning in a cadaveric study. We hypothesized that this new technology could be reliably used, providing high accuracy of implant position in a cadaveric setting.

\section{Methods}

\section{Ethical statement}

The study has been performed in accordance with the ethical standards in the 1964 Declaration of Helsinki. The study was accepted by the cantonal ethics committee of Zurich under the number 2017-00874. All experiments were conducted in a human cadaveric laboratory, access was granted only to people directly involved in the experiments.

\section{Study design}

The study was conducted with one group of twelve freshfrozen human cadaveric scapulae with the adjacent humerus. According to the planning, one orthopedic surgeon (P.K.) placed one guidewire for baseplate positioning in each glenoid under AR navigation. The planning and analysis were done using computed tomography.

\section{Experimental procedures}

The experimental procedures were all conducted in a standardized setting at the same time of the day on six consecutive days. All twelve deep-frozen cadavers were scanned using computed tomography (Siemens Somotom Edge Plus, Germany) in $0.5 \mathrm{~mm}$ slice increments. Each scapular was segmented separately with global thresholding and the region growing tool in a standard segmentation software (MIMICS 23, Leuven, Belgium). The 3D models were exported as *.stl-Files and imported into our institution's standard 3D-Planning software (CASPA, Balgrist CARD, Zurich, Switzerland). The guidewire position was planned in the computer software to reach inferior baseplate position in neutral version and inclination. The data were then prepared and converted using Unity Software (Unity Technologies, San Francisco, CA, USA, Version, 2019.1.7) and Microsoft Visual Studio (version Community 2017, Microsoft Corporation, Redmond, WA, USA) and then set up on the HoloLens.

Each scapula was placed in a self-manufactured holding device at the institution's cadaver laboratory. The human cadaveric models contained the full scapula and humeral bone with all adjacent muscles, soft tissue, and skin to simulate natural conditions. For all individuals, a deltopectoral approach was utilized. The subscapularis muscle was sharply dissected at the lesser tuberosity and held out of the operative field with retaining cords. The supraspinatus muscle was slightly dissected at the footprint and kept dorsally. Then the joint capsule was resected. Subsequently, the humerus could be dorsally sub-luxated and held away using retractors. The glenoid came into full view.

Then the navigation was started. A $1.6 \mathrm{~mm}$ Kirschnerwire was placed with a drilling machine (PSR14,4 LI-2, Bosch AG, Gerlingen, Germany) in every glenoid using Microsoft HoloLense for augmented reality navigation through a head-mounted display (described below).

The achieved Kirschner-wire position was analyzed using a second computed tomography. The scanned scapulae were segmented as described above and imported into our institution's planning software (CASPA, Balgrist CARD, Zurich, Switzerland) to overlay the preoperative and postoperative scapula by nearest iterative point cloud analysis [14]. The deviation from the planning (entry point in $\mathrm{mm}$ and trajectory in a three-dimensional angle error) was calculated.

\section{Surgical navigation}

The Microsoft Head-Mounted Display HoloLense Version 1 (Microsoft, Redmond, WA, USA) was employed 
in the study to register the position of the scapula in the room, to set up a hologram exactly over the operative site to navigate the guidewire. Voice commands and gestures controlled the HoloLense.

The scapula position was registered using a custom-made fiducial marker and stereo-tracking, as described before [15] (Fig. 1). First, the rough orientation was defined through the location of the acromion, coracoid, and glenoid. Those points were defined in the preoperative planning using our institution's software (CASPA). Second, the fine adjustment was achieved by tracing the glenoids' surface. The exact location of the hologram was then calculated and superimposed as augmented reality to the operative site. After confirming the optimal position, the navigation process was started (Fig. 2, Video 1). A virtual model of the scapula appeared in the surgeon's field of vision. The drill sleeve with a fiducial marker was used to display the planned entry point with deviation in millimeters and the planned vector
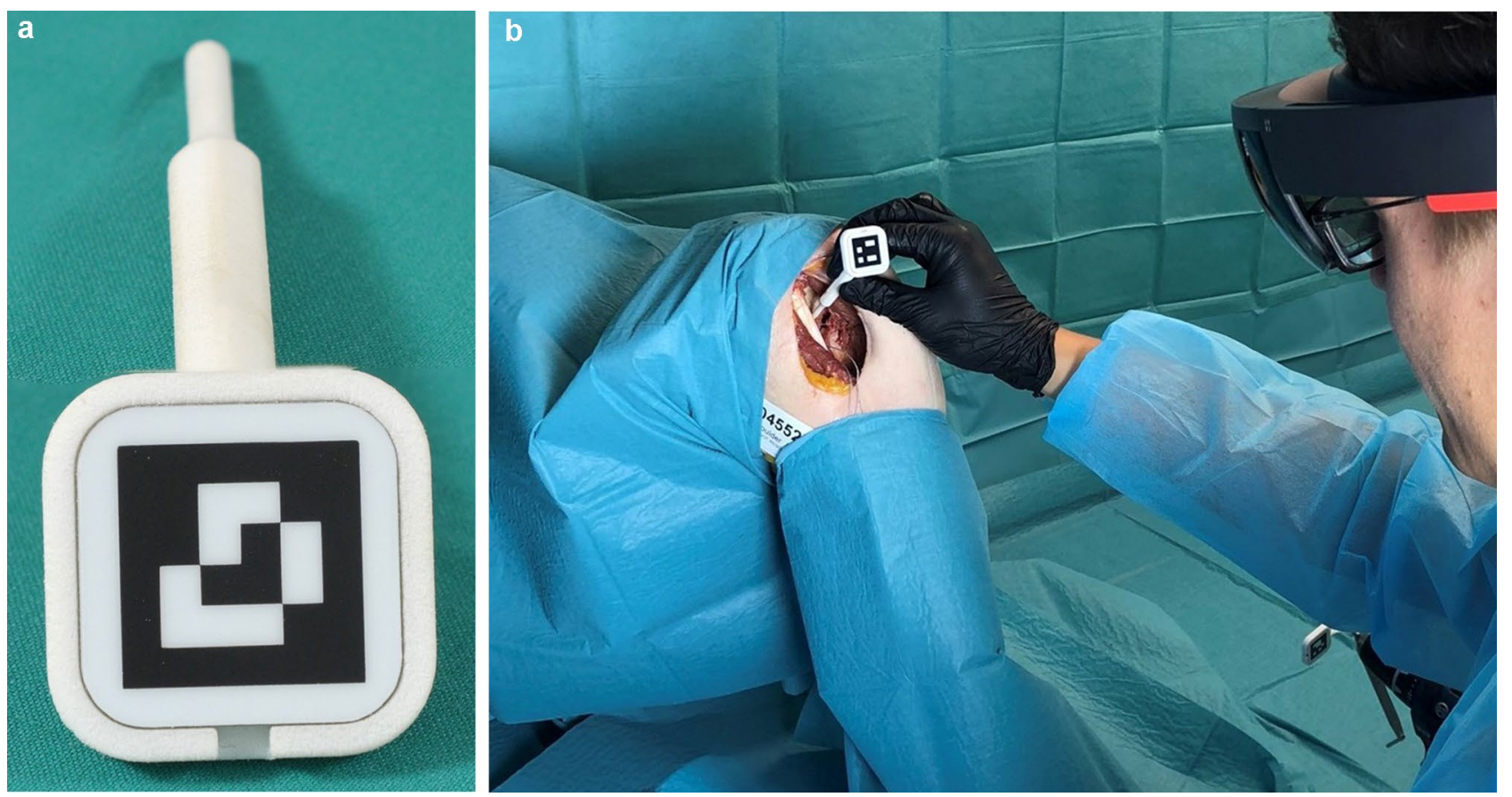

Fig. 1 The registration process necessary to achieve hologram overlay. a The fiducial marker to detect and track the surface. b The intraoperative application
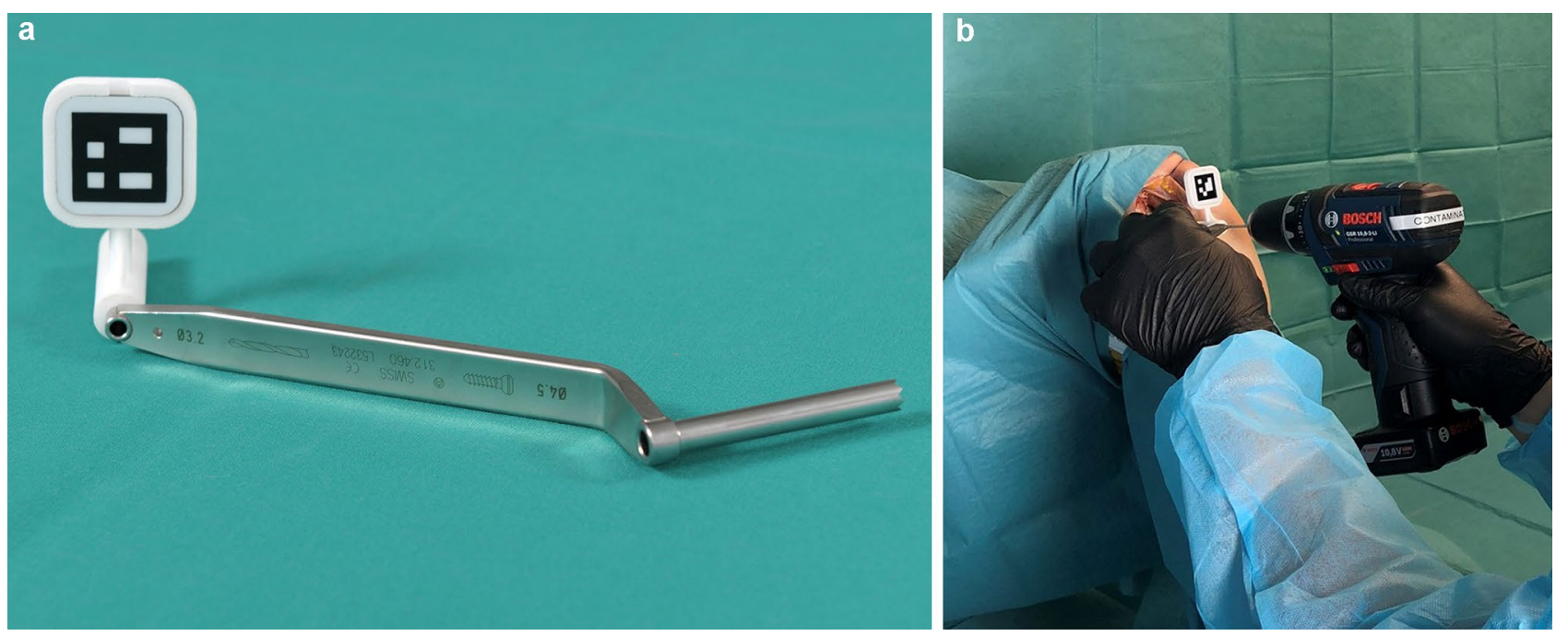

Fig. 2 The navigation process with the a drill guide for head-mounted display navigation. b The intraoperative application 


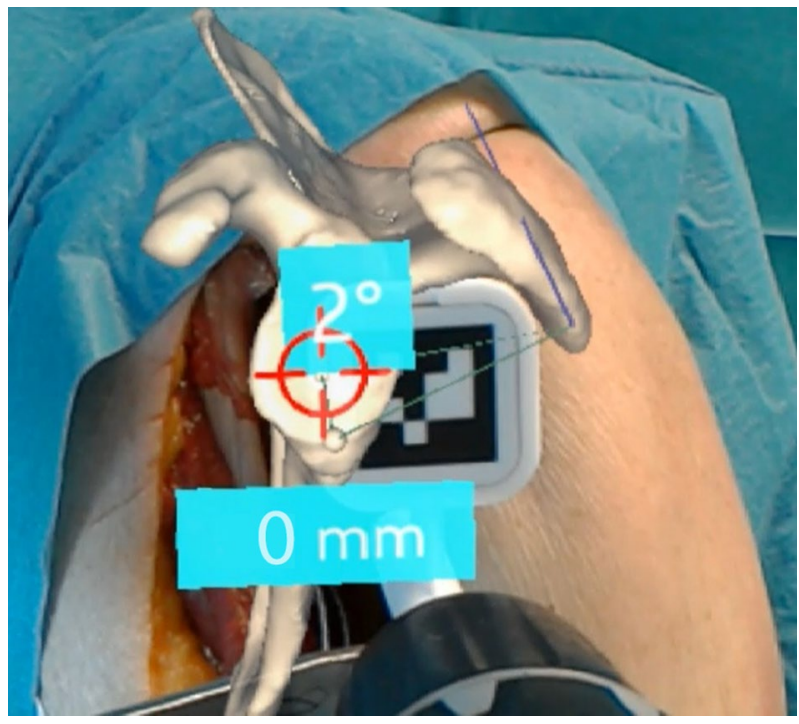

Fig. 3 The surgeon's view and is obtained from the head-mounted display camera. The superimposed hologram shows the scapula with the planned entry point and the deviation in millimeters. The planned trajectory of the guidewire and the current deviation is displayed in degrees

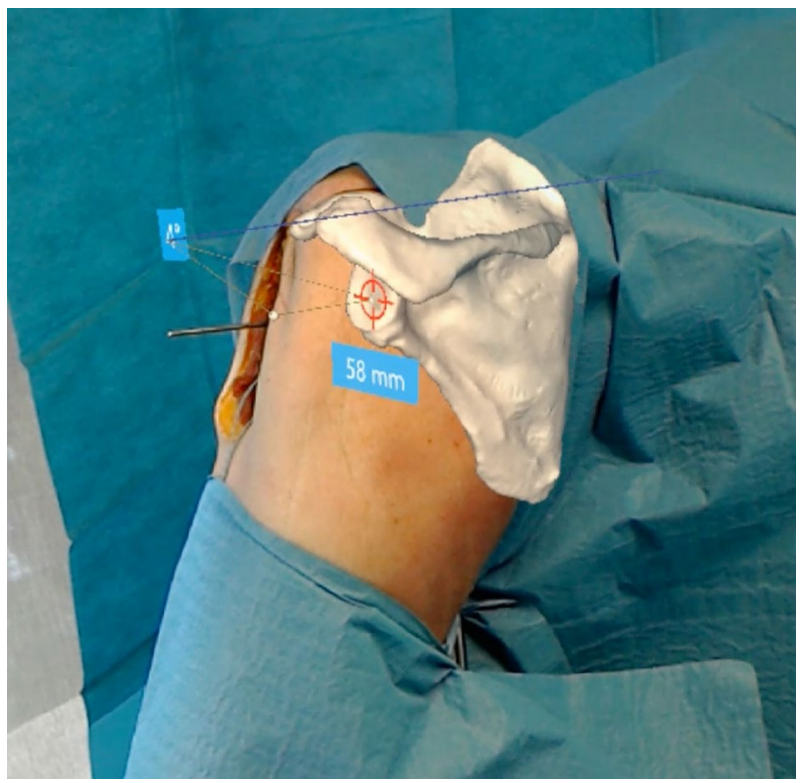

Fig. 4 The surgeon's navigation perspective from another angle. The scapula is permanently superimposed on the real anatomy after registration. This allows viewing from several angles

with deviation in degree (Fig. 3). With this information, the target wire could be placed (Fig. 4).

\section{Outcome parameters}

Outcome measures were deviation from the planned guidewire direction (vector), deviation of the glenoid side entry point, and the number of attempts to get optimal hologram overlay.

The 3D angular error (AE) was calculated using the direction vector of the planned $(\vec{A})$ and executed $(\vec{B})$ trajectories by applying the following formula:

$\mathrm{AE}=\cos ^{-1}\left(\frac{\vec{A} \cdot \vec{B}}{\|\vec{A}\|\|\vec{B}\|}\right)$

The entry point error (TE) was calculated as Euclidean distance between the centers of the planned $\left(x_{1}, y_{1}, z_{1}\right)$ and achieved $\left(x_{2}, y_{2}, z_{2}\right)$ entry points using the following formula:

$\mathrm{TE}=\sqrt{\left(x_{1}-x_{2}\right)^{2}+\left(y_{1}-y_{2}\right)^{2}+\left(z_{1}-z_{2}\right)^{2}}$

The analysis is shown in Fig. 5.

Adverse events were recorded. They were defined as technical or patient-specific events. Technical issues included problems recognizing the scapula or the tracker by the Holo Lense or the impossibility of navigating the guidewire. Patient-specific complications included intraoperative fractures during drilling or damage to critical structures such as nerves or vessels.

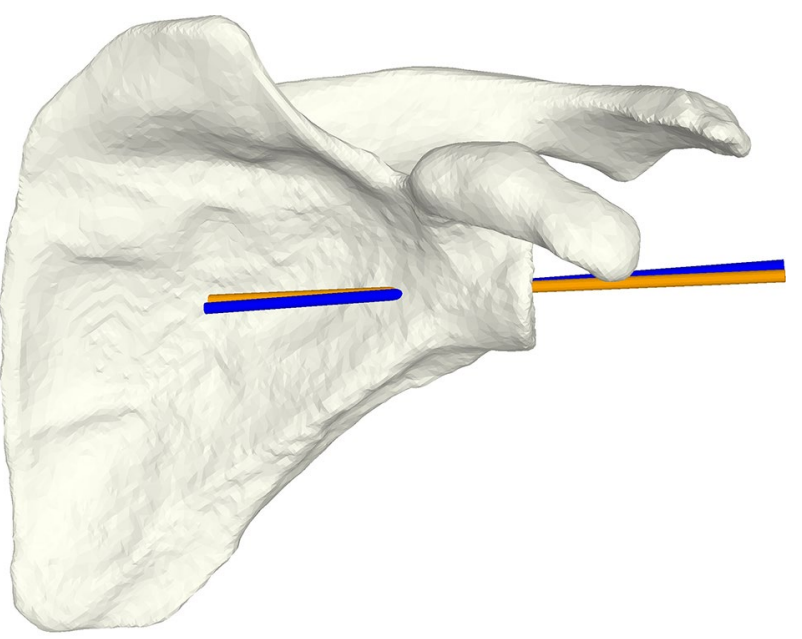

Fig. 5 Postoperative calculation of achieved deviation. Preoperative planning is represented by the orange vector, which was planned after the first $\mathrm{CT}$ imaging. The blue vector corresponds to the intraoperatively placed wire, determined by second CT imaging 


\section{Statistical analysis}

For statistical calculations, SPSS v23.0 (IBM, New York, United States of America) was utilized. Descriptive statistics are given as mean \pm standard deviation (95\% confidence interval). Comparison of nonparametric data was performed using the Mann-Whitney $U$ test.

\section{Results}

We included all twelve shoulders in our analysis. The mean age was $73 \pm 2(71-75)$ years, eight of twelve shoulders were male with four right and eight left shoulders.

All twelve guidewires were placed without any technical problems. The software worked without problems. Only one registration attempt to overlay the image correctly was necessary for eleven of twelve specimens, and two attempts were required for the other specimen. The mean deviation from the planned entry point was $3.5 \mathrm{~mm} \pm 1.7 \mathrm{~mm}(95 \%$ CI $2.4 \mathrm{~mm} ; 4.6 \mathrm{~mm})$. The mean deviation from the planned trajectory was $3.8^{\circ} \pm 1.7^{\circ}\left(95 \%\right.$ CI $\left.2.6^{\circ} ; 4.9^{\circ}\right)$ (Table 1$)$.

The side ( 8 left shoulders, 4 right shoulders) did not influence the deviation from the planned vector $(p<0.46)$ and planned entry point $(p=1.0)$ in the setting of a right-handed surgeon. There was no subjective difference in treatment of right or left shoulders for the surgeon using HMD-assisted navigation. No adverse event occurred.

Table 1 Results of the baseplate navigation accuracy using augmented reality through head-mounted display

\begin{tabular}{lllll}
\hline ID & 3D vector & Entry point & Attempts & Side \\
\hline 1 & $1.0^{\circ}$ & $3.3 \mathrm{~mm}$ & 1 & Left \\
2 & $3.2^{\circ}$ & $5.7 \mathrm{~mm}$ & 1 & Left \\
3 & $7.8^{\circ}$ & $2.3 \mathrm{~mm}$ & 2 & Left \\
4 & $3.2^{\circ}$ & $4.3 \mathrm{~mm}$ & 1 & Right \\
5 & $3.4^{\circ}$ & $4.0 \mathrm{~mm}$ & 1 & Right \\
6 & $4.7^{\circ}$ & $0.6 \mathrm{~mm}$ & 1 & Left \\
7 & $4.3^{\circ}$ & $2.3 \mathrm{~mm}$ & 1 & Left \\
8 & $4.9^{\circ}$ & $5.4 \mathrm{~mm}$ & 1 & Left \\
9 & $4.7^{\circ}$ & $1.9 \mathrm{~mm}$ & 1 & Left \\
10 & $3.4^{\circ}$ & $4.0 \mathrm{~mm}$ & 1 & Right \\
11 & $1.0^{\circ}$ & $6.2 \mathrm{~mm}$ & 1 & Left \\
12 & $3.3^{\circ}$ & $1.6 \mathrm{~mm}$ & 1 & Right \\
Mean & $3.8^{\circ} \pm 1.7^{\circ}$ & $3.5 \pm 1.7 \mathrm{~mm}$ & $1 \pm 0.3$ & \\
$95 \% \mathrm{CI}$ & $\left(95 \% \mathrm{CI} 2.6^{\circ} ; 4.9^{\circ}\right)$ & $(95 \% \mathrm{CI} 2.4 ; 4.6)$ & & \\
\hline
\end{tabular}

The values $3 \mathrm{D}$ vector and entry point describe the deviation from the planning measured by comparing the preoperatively planned vector with the postoperatively achieved vector using computed tomography. Attempts reflect the number of registration processes to achieve adequate hologram overlay

CI confidence interval, $\mathrm{mm}$ millimeter

\section{Discussion}

The conducted study confirmed our hypotheses that (1) the use of AR navigation to position the glenoid baseplate component in RSA is feasible and (2) can achieve good accuracy in a cadaveric setting. The mean deviation from the planned entry point was $3.5 \mathrm{~mm}$ and the deviation from the planned 3D trajectory was $3.8^{\circ}$. We saw high reliability with mostly one registration attempt necessary to achieve correct hologram superimposition.

To the best of our knowledge, this is the first report in the current literature that describes glenoid guidewire positioning utilizing AR with an HMD as a navigation device in a cadaveric model. We previously analyzed the use of AR in ten 3D-printed scapulae and found a mean deviation from the entry point of $2.26 \mathrm{~mm} \pm 1.11 \mathrm{~mm}$. The deviation from the planned vector was $2.74^{\circ} \pm 1.25^{\circ}$ [13] Berhouet et al. described the application of augmented reality to display a three-dimensional reconstruction of the glenoid and the adjacent scapula using a head-mounted display [11]. On a technical note, Gregory et al. described using a head-mounted display intraoperatively in one single patient. They could drag and drop the scapula in the operative field, display the computed tomography data, and conference with other surgeons remotely [12].

Our study results should be compared to other AR applications. Wang et al. [16] described AR through HMD for navigation of pedicle screws with a deviation trajectory of $2.9^{\circ} \pm 1.1^{\circ}$ and a deviation from the entry point of $2.7 \pm 1.2 \mathrm{~mm}$ in 6 human cadavers. Müller et al. [17] tested the AR navigation through a head-mounted display for pedicle screw instrumentation in human cadavers. The results were comparable to a state-of-the-art posetracking system. Molino et al. [18] described in another human cadaver study non-inferiority of AR using a HMD in comparison to freehand, manual computer-navigated, and robotics-assisted computer-navigated pedicle screw placement. Other studies in the current literature reported mainly using HMD to show information in the clinical or cadaveric setting, less on the function of AR navigation through HMD. When used as a replacement for a freestanding monitor, the duration of surgery could be shortened with a lower radiation dose and increased concentration on the surgical area $[19,20]$.

The optimal application area for HMD-navigated component placement has yet to be defined. Currently, especially for severe glenoid deformities, PSI or computer-supported pose tracking systems are well-validated systems. A recently published meta-analysis of 277 shoulders showed a $2 \mathrm{D}$ accuracy of $2.7^{\circ} \pm 0.5^{\circ}$ for the version and $1.9^{\circ} \pm 0.4^{\circ}$ for the inclination with a deviation of the entry point of $1.1 \mathrm{~mm} \pm 0.2 \mathrm{~mm}$ when using PSI. This was superior to 
the standard freehand method (version $5.9^{\circ} \pm 1.1^{\circ}$, inclination $5.8^{\circ} \pm 1^{\circ}$, entry point $2 \mathrm{~mm} \pm 0.4 \mathrm{~mm}$ ) [8]. Another recently published meta-analysis from 2019 including seven studies revealed a mean deviation from the planning of $3.2^{\circ}\left(95 \% \mathrm{CI}-4.6^{\circ}\right.$ to $\left.+4.6^{\circ}\right)$ and $1.2^{\circ}\left(95 \% \mathrm{CI}-4.6^{\circ}\right.$ to $+1.3^{\circ}$ ) for version and inclination, respectively. The offset deviation was $0.2 \mathrm{~mm}$ ( $95 \% \mathrm{CI}-4.6$ to $0.4 \mathrm{~mm}$ ). Since they included only studies comparing PSI to freehand, no statistically significant difference was detected, albeit the deviation for freehand baseplate positioning was larger [21]. The disadvantages of PSI are the costs and long preparation time required for the individual planning and production of the guides. AR through HMD offers a straightforward application in the operating room with low costs for the setup.

Computer navigation using pose-tracking reaches similar results but can be complex and error-prone in the setup. Stübig et al. compared 15 navigated and 12 conventional glenoid baseplate implantations and detected no difference for inclination, but a significantly better version with $1.6^{\circ} \pm 4.5^{\circ}$ and $11.5^{\circ} \pm 6.5^{\circ}$ for the navigated and conventional group, respectively [22]. Wang described 25 prospectively followed cases with a mean deviation from the planing for version of $3^{\circ} \pm 2^{\circ}$ and for inclination of $5^{\circ} \pm 3^{\circ}[23]$.

The presented results of PSI and pose-tracking navigation are comparable to our study results. A further development of navigation using AR through HMD should be promoted, knowing better hardware will be implemented.

Several limitations have to be discussed. (1) It was a human cadaver study without in vivo problems like bleeding, the setting of an operation room, or the movement of the chest and scapula during breathing. (2) The time necessary to navigate the guidewires was not measured. This fact was accepted knowingly, as the study aimed to analyze the feasibility and accuracy in a cadaveric setting. In the study planning, time recording was rejected to avoid influence on results by overhasty insertion of the wires. Nevertheless, a surprisingly low time expenditure can be reported as a maximum of two registration attempts were necessary to overlay the hologram in each individual. (3) We decided to conduct the study without a control group to test the proposed navigation method against the optimal position, calculating the deviation from the planned entry point and planned trajectory. Future studies are mandatory to prove the superiority of this navigation system over freehand guidewire positioning, ideally performed by multiple surgeons. In the further course, an application should be implemented in clinical studies and a functional extension for additional placement of the glenoid screws at the site of maximum bone strength.

The results of this study have to be interpreted accepting the original purpose of Microsoft's Holo Lense as multimedia and entertaining device. Primarily, it was not invented to function as a medical device with a high necessity of accuracy and reliability. Nevertheless, a valuable and reliable application could be shown in this study. Upcoming AR devices will probably be designed to serve medical purposes and can thus be equipped with high-end and eventually more expensive hardware.

\section{Conclusion}

Baseplate navigation in reverse total shoulder arthroplasty using augmented reality through a head-mounted display seems feasible, showing high accuracy in a cadaveric shoulder model.

Supplementary Information The online version contains supplementary material available at https://doi.org/10.1007/s00402-021-04025-5.

Acknowledgements This work is part of "SURGENT" under the umbrella of University Medicine Zurich/Hochschulmedizin Zürich. Cadaveric studies were conducted in the human surgery laboratories at Balgrist Campus AG, Zürich. Imaging was performed with the support of the Swiss Center for Musculoskeletal Imaging, SCMI, Balgrist Campus AG, Zurich. We thank Christian Streng for filming and movie editing and Florentin Liebmann for technical and software support. We thank the Balgrist CARD and the Incremed AG for their great support.

Funding Open Access funding provided by Universität Zürich. The study was funded internally by the orthopaedic department.

\section{Declarations}

Conflict of interest Each author certifies that he or she has no commercial associations (e.g., consultancies, stock ownership, equity interest, patent/licensing arrangements, etc.) that might pose a conflict of interest in connection with the submitted article.

Ethical approval IRB: This study was performed in line with the principles of the Declaration of Helsinki. Approval was granted by the Ethics Committee of the University Zurich (2017-00874).

Open Access This article is licensed under a Creative Commons Attribution 4.0 International License, which permits use, sharing, adaptation, distribution and reproduction in any medium or format, as long as you give appropriate credit to the original author(s) and the source, provide a link to the Creative Commons licence, and indicate if changes were made. The images or other third party material in this article are included in the article's Creative Commons licence, unless indicated otherwise in a credit line to the material. If material is not included in the article's Creative Commons licence and your intended use is not permitted by statutory regulation or exceeds the permitted use, you will need to obtain permission directly from the copyright holder. To view a copy of this licence, visit http://creativecommons.org/licenses/by/4.0/.

\section{References}

1. Schairer WW, Nwachukwu BU, Lyman S, Craig EV, Gulotta LV (2015) National utilization of reverse total shoulder arthroplasty 
in the United States. J Shoulder Elbow Surg 24(1):91-97. https:// doi.org/10.1016/j.jse.2014.08.026

2. Kim SH, Wise BL, Zhang Y, Szabo RM (2011) Increasing incidence of shoulder arthroplasty in the United States. J Bone Joint Surg Am 93(24):2249-2254. https://doi.org/10.2106/JBJS.J. 01994

3. Day JS, Lau E, Ong KL, Williams GR, Ramsey ML, Kurtz SM (2010) Prevalence and projections of total shoulder and elbow arthroplasty in the United States to 2015. J Shoulder Elbow Surg 19(8):1115-1120. https://doi.org/10.1016/j.jse.2010.02.009

4. Edwards TB, Trappey GJ, Riley C, O'Connor DP, Elkousy HA, Gartsman GM (2012) Inferior tilt of the glenoid component does not decrease scapular notching in reverse shoulder arthroplasty: results of a prospective randomized study. J Shoulder Elbow Surg 21(5):641-646. https://doi.org/10.1016/j.jse.2011.08.057

5. Nyffeler RW, Sheikh R, Atkinson TS, Jacob HA, Favre P, Gerber $C$ (2006) Effects of glenoid component version on humeral head displacement and joint reaction forces: an experimental study. J Shoulder Elbow Surg 15(5):625-629. https://doi.org/10.1016/j. jse.2005.09.016

6. Gutierrez S, Walker M, Willis M, Pupello DR, Frankle MA (2011) Effects of tilt and glenosphere eccentricity on baseplate/ bone interface forces in a computational model, validated by a mechanical model, of reverse shoulder arthroplasty. J Shoulder Elbow Surg 20(5):732-739. https://doi.org/10.1016/j.jse.2010.10. 035

7. Zumstein MA, Pinedo M, Old J, Boileau P (2011) Problems, complications, reoperations, and revisions in reverse total shoulder arthroplasty: a systematic review. J Shoulder Elbow Surg 20(1):146-157. https://doi.org/10.1016/j.jse.2010.08.001

8. Villatte G, Muller AS, Pereira B, Mulliez A, Reilly P, Emery R (2018) Use of Patient-Specific Instrumentation (PSI) for glenoid component positioning in shoulder arthroplasty. A systematic review and meta-analysis. PLoS ONE 13(8):e0201759. https:// doi.org/10.1371/journal.pone.0201759

9. Sadoghi P, Vavken J, Leithner A, Vavken P (2015) Benefit of intraoperative navigation on glenoid component positioning during total shoulder arthroplasty. Arch Orthop Trauma Surg 135(1):41-47. https://doi.org/10.1007/s00402-014-2126-1

10. Jud L, Fotouhi J, Andronic O, Aichmair A, Osgood G, Navab N, Farshad M (2020) Applicability of augmented reality in orthopedic surgery-a systematic review. BMC Musculoskelet Disord 21(1):103. https://doi.org/10.1186/s12891-020-3110-2

11. Berhouet J, Slimane M, Facomprez M, Jiang M, Favard L (2019) Views on a new surgical assistance method for implanting the glenoid component during total shoulder arthroplasty. Part 2: from three-dimensional reconstruction to augmented reality: feasibility study. Orthop Traumatol Surg Res 105(2):211-218. https://doi. org/10.1016/j.otsr.2018.08.021

12. Gregory TM, Gregory J, Sledge J, Allard R, Mir O (2018) Surgery guided by mixed reality: presentation of a proof of concept. Acta Orthop 89(5):480-483. https://doi.org/10.1080/17453674.2018. 1506974
13. Kriechling P, Roner S, Liebmann F, Casari F, Furnstahl P, Wieser K (2020) Augmented reality for base plate component placement in reverse total shoulder arthroplasty: a feasibility study. Arch Orthop Trauma Surg. https://doi.org/10.1007/s00402-020-03542-z

14. Besl PJ, McKay ND (1992) A method for registration of 3-D shapes. IEEE Trans Pattern Anal Mach Intell 14(2):239-256. https://doi.org/10.1109/34.121791

15. Liebmann F, Roner S, von Atzigen M, Scaramuzza D, Sutter R, Snedeker J, Farshad M, Furnstahl P (2019) Pedicle screw navigation using surface digitization on the Microsoft HoloLens. Int J Comput Assist Radiol Surg 14(7):1157-1165. https://doi.org/10. 1007/s11548-019-01973-7

16. Wang H, Wang F, Leong AP, Xu L, Chen X, Wang Q (2016) Precision insertion of percutaneous sacroiliac screws using a novel augmented reality-based navigation system: a pilot study. Int Orthop 40(9):1941-1947. https://doi.org/10.1007/s00264-015-3028-8

17. Muller F, Roner S, Liebmann F, Spirig JM, Furnstahl P, Farshad M (2020) Augmented reality navigation for spinal pedicle screw instrumentation using intraoperative $3 \mathrm{D}$ imaging. Spine $\mathrm{J}$ 20(4):621-628. https://doi.org/10.1016/j.spinee.2019.10.012

18. Molina CA (2020) Erratum. Augmented reality-assisted pedicle screw insertion: a cadaveric proof-of-concept study. J Neurosurg Spine. https://doi.org/10.3171/2020.3.SPINE181142a

19. Ortega G, Wolff A, Baumgaertner M, Kendoff D (2008) Usefulness of a head mounted monitor device for viewing intraoperative fluoroscopy during orthopaedic procedures. Arch Orthop Trauma Surg 128(10):1123-1126. https://doi.org/10.1007/ s00402-007-0500-y

20. Yoon JW, Chen RE, Han PK, Si P, Freeman WD, Pirris SM (2017) Technical feasibility and safety of an intraoperative headup display device during spine instrumentation. Int J Med Robot 13(3):e1770. https://doi.org/10.1002/rcs.1770

21. Cabarcas BC, Cvetanovich GL, Gowd AK, Liu JN, Manderle BJ, Verma NN (2019) Accuracy of patient-specific instrumentation in shoulder arthroplasty: a systematic review and meta-analysis. JSES Open Access 3(3):117-129. https://doi.org/10.1016/j.jses. 2019.07.002

22. Stubig T, Petri M, Zeckey C, Hawi N, Krettek C, Citak M, Meller $\mathrm{R}$ (2013) 3D navigated implantation of the glenoid component in reversed shoulder arthroplasty. Feasibility and results in an anatomic study. Int J Med Robot 9(4):480-485. https://doi.org/10. 1002/rcs. 1519

23. Wang AW, Hayes A, Gibbons R, Mackie KE (2020) Computer navigation of the glenoid component in reverse total shoulder arthroplasty: a clinical trial to evaluate the learning curve. J Shoulder Elbow Surg 29(3):617-623. https://doi.org/10.1016/j.jse.2019. 08.012

Publisher's Note Springer Nature remains neutral with regard to jurisdictional claims in published maps and institutional affiliations. 